\title{
Osteosarcoma Journey in India: Each Step Reveals a New Horizon!
}

Osteosarcoma remains one of the most challenging cancers of childhood and young adults wherein the addition of chemotherapy to surgery has led to dramatic but improvements in the event-free survival (EFS) (from 20\% to $60 \%$ ). However, these improvements have been largely reported in developed nations, with a gross mismatch seen in low- and middle-income countries (LMICs). However, even in high-income countries, the outcomes are largely static since the past three decades. ${ }^{[1]}$

Osteosarcoma therapy protocols mainly utilize a dyad backbone of cisplatin and doxorubicin, with the addition of a third drug, either ifosfamide or high-dose methotrexate (HDMTX), which has led to rapid strides forward in survival in osteosarcoma. ${ }^{[1]}$

HDMTX-based regimen is largely used in the developed world as a custom. However, there is no randomized proof of the superiority of this regimen over a non-HDMTX-based regimen. ${ }^{[2]}$

In LMICs, where the bulk of the patients present with malnutrition and advanced disease, ${ }^{[3]}$ the feasibility of HDMTX is called into question. The in-patient administration requirements, complex drug levels monitoring, and stringent hydration control, with increased nursing care, ${ }^{[4]}$ are significant hurdles, other than costs and unpredictable toxicity. Further, the fertility-sparing potential of HDMTX-based regimen is largely negated by the concomitant use of mandatory alkylator (cisplatin).

Considering the poor ratio of both beds as well as nurses to patients in Tata Memorial Center, sustainable options were sorted over two decades which could be implemented without burdening the existing systems. Thus, non-HDMTX protocols were conceptualized and over the course of many years, the protocols were fine-tuned using principles of dose-density and tumor kinetics. The first protocol used was the OGS-99 protocol implemented in the year 2000 consisting of ifosfamide, doxorubicin, and cisplatin administered together as three cycles before and after surgery. The 5-year EFS for this protocol was suboptimal (38\%) and the push for improvement continued. ${ }^{[5]}$

In 2010, the OGS-99-enhanced protocol was devised which added etoposide to the existing drugs and consisted of a total of nine cycles of chemotherapy. Compliance of patients was found to be an important factor in improving survival. This was the first milestone achieved by proper counseling and patient education. This protocol also incorporated the use of prophylactic granulocyte-colony-stimulating factor (G-CSF), as the generic versions were available, bringing down costs. ${ }^{[6]}$ These measures improved the outcomes resulting in an EFS of $50 \%$ and overall survival (OS) of $60 \% .{ }^{[5]}$ However, the road to improvement did not end there.

In late 2011, the present protocol, "OGS-12," was designed. $^{[3]}$ It consisted of four cycles of neoadjuvant chemotherapy and four cycles of adjuvant chemotherapy. The three most potent drugs were used doxorubicin, ifosfamide, and cisplatin administered in a sequential fashion to increase the dose density. Etoposide was omitted, being the least effective with only added toxicity. ${ }^{[3]}$ Cycles 1 and 2 comprised doxorubicin (25 mg/m $\mathrm{m}^{2}$ days $\left.1-3\right)$ and cisplatin (33 mg/m² days $1-3)$ and cycles 3 and 4 comprised ifosfamide $\left(1.8 \mathrm{~g} / \mathrm{m}^{2}\right.$ days $\left.1-5\right)$ and doxorubicin in the same dose. Cycles 5, 6, 7, and 8 comprised ifosfamide and cisplatin. Cumulative doses of the drugs were doxorubicin $300 \mathrm{mg} / \mathrm{m}^{2}$, cisplatin $600 \mathrm{mg} / \mathrm{m}^{2}$, and ifosfamide $54 \mathrm{~g} / \mathrm{m}^{2}$. All patients received G-CSF prophylaxis for 7 days. Nutritional deficiencies were looked for and corrected using iron and folate supplementation. Patients were closely followed up for chemotoxicities with serial echocardiograms, diethylenetriaminepentaacetic acid-glomerular filtration rate measurements, audiometry, and corrective measures taken. Patients were again counseled regarding compliance and side effects, and they were followed up continually to ensure adherence to treatment. The outcomes of 385 patients who were given this protocol were analyzed, and all the above interventions helped us to improve the 5 -year EFS to $62 \%$ and OS to $77 \%{ }^{[5]}$ The improvement in survival can be clearly attributed to the application of the principles of chemotherapy dosing and tumor response ${ }^{[6]}$ along with the enhanced supportive care and follow-up of patients. The 3-year survival using a non-HDMTX protocol in osteosarcoma patients was $54.6 \%$ in a South Indian study. ${ }^{[7]}$ Successive studies in another tertiary care center demonstrated 32\% good responders and a 5-year survival of $50 \%$ treated with a protocol without HDMTX. ${ }^{[8-10]}$

The "OGS-12" protocol was well tolerated with comparable toxicities. The incidence of febrile neutropenia (FN) was $40 \%$ with three chemotherapy-related toxicity deaths. Two of the patients developed secondary hematological malignancies during follow-up. Cardiac, renal, and ototoxicity were not significant. The protocol was relatively easy to administer in an outpatient setting and did not require the rigorous monitoring that HDMTX entailed.

Some of the factors that correlated with survival were good performance status, timely completion of the entire protocol, good histological response, female gender, and occurrence of FN. The atypical correlation of female gender with survival may be due to the link between cancer outcomes, the circadian system, and varied gender-specific 
clock-controlled gene expression. ${ }^{[9]}$ Patients developing FN are probably more chemoresponsive and have additional immune-mediated antitumor activity. Historically, William Coley, using toxins, proved that infections can induce remissions in sarcoma. ${ }^{[10]}$ It is known that the infection generates inflammatory factors that activate resting dendritic cells and T-cells that target the tumor. ${ }^{[1]}$

The overall cost of the "OGS-12" treatment was considerably lower compared to the methotrexate, Adriamycin, and platinum (HDMTX) regimen ${ }^{[12]}$ used in the Western world (2100\$ vs. $7000 \$) \cdot{ }^{[5]}$ This protocol is of immense value, especially in LMICs where health systems are overburdened along with various cost constraints and patients presenting with very advanced disease. Addressing inefficiencies today is a vital measure to improve the quality of cancer care and to continuously evolve, we may require some "disruptive changes." Sometimes, seemingly "simple" practices may significantly impact patient care, as is evident by the evolution of the management of osteosarcoma.

Studies are ongoing regarding the use of immunotherapy in osteosarcoma as well as the targeting biological pathways such as the $\mathrm{PI} 3 \mathrm{~K} / \mathrm{mTOR}$, WNT/Bcatenin, transforming growth factor beta, receptor activator of nuclear factor-kappa B ligand/nuclear factor kappa B, and insulin-like growth factor. These new molecules in addition to chemotherapy may further enhance outcomes of osteosarcoma patients. ${ }^{[13-15]}$

The novel "OGS-12" protocol demonstrates how careful planning and thoughtful interventions like identifying and correcting deficiencies can improve on existing systems leading to improved patient survival and easier implementation. The journey does not end here, though, and we hope to build on this foundation and further better the protocol based on newer evidence and principles. Each step will take us toward the goal of curing every patient with nonmetastatic osteosarcoma!

\section{Jyoti Bajpai ${ }^{1,2}$, Arun Chandrashekharan ${ }^{3}$, Shripad D Banavali ${ }^{4}$, Sudeep Gupta ${ }^{1,2}$}

${ }^{1}$ Tata Memorial Centre, Tata Memorial Hospital, Mumbai, Maharashtra, India, ${ }^{2}$ Advanced Centre for Treatment Research and Education in Cancer, Tata Memorial Centre, Homi Bhabha National Institute, Kharghar, Navi Mumbai, Maharashtra, India, ${ }^{3}$ Department of Medical Oncology, Aster Malabar Institute of Medical Sciences, Kozhikode, Kerala, India, ${ }^{4}$ Department of Medical and Pediatric Oncology, TMC-Rural Outreach Program, Tata Memorial Centre, Tata Memorial Hospital, Homi Bhabha National Institute, Mumbai, Maharashtra, India
Address for correspondence: Dr. Jyoti Bajpai, Tata Memorial Centre, Tata Memorial Hospital, Dr. Ernest Borges Marg, Parel, Mumbai - 400 012, Maharashtra, India. Advanced Centre for Treatment Research and Education in Cancer, Tata Memorial Centre, Homi Bhabha National Institute, Kharghar, Navi Mumbai - 410 210, Maharashtra, India. E-mail:dr_jyotibajpai@yahoo.co.in

Submitted: 17-Jan-2020

Revised: 28-Jan-2020

Accepted: $21-$ Feb-2020

Published: 24-Apr-2020

\section{References}

1. Bajpai J, Jaffe N. Perspectives of the role of chemotherapy in the management of osteosarcoma. JCT 2012;03:1191-203.

2. Souhami RL, Craft AW, van der Eijken JW, Nooij M, Spooner D, Bramwell $\mathrm{VH}$, et al. Randomised trial of two regimens of chemotherapy in operable osteosarcoma: A study of the European Osteosarcoma Intergroup. Lancet 1997;350:911-7.

3. Bajpai J, Chandrasekharan A, Talreja V, Simha V, Chandrakanth MV, Rekhi B, et al. Outcomes in non-metastatic treatment naive extremity osteosarcoma patients treated with a novel non-high dosemethotrexate-based, dose-dense combination chemotherapy regimen 'OGS-12'. Eur J Cancer 2017;85:49-58.

4. Graf N, Winkler K, Betlemovic M, Fuchs N, Bode U. Methotrexate pharmacokinetics and prognosis in osteosarcoma. J Clin Oncol 1994;12:1443-51.

5. Bajpai J, Chandrasekharan A, Simha V, Mandal T, Shah K, Hingmare $\mathrm{S}$, et al. Osteosarcoma journey over two decades in India: Small steps, big changes. Pediatr Blood Cancer 2019;66:e27877.

6. Norton L, Simon R. Tumor size, sensitivity to therapy, and design of treatment schedules. Cancer Treat Rep 1977;61:1307-17.

7. Sukumaran RK, Rajeshwari B, Sugath S, Chellappan SG, Thankamony P, Parukuttyamma K. Methotrexate free chemotherapy and limb salvage surgery for paediatric osteosarcoma in India. Indian J Orthop 2018;52:58-64.

8. Bajpai J, Sharma M, Sreenivas V, Kumar R, Gamnagatti S, Khan SA, et al. VEGF expression as a prognostic marker in osteosarcoma. Pediatr Blood Cancer 2009;53:1035-9.

9. Nataraj V, Batra A, Rastogi S, Khan SA, Sharma MC, Vishnubhatla S, et al. Developing a prognostic model for patients with localized osteosarcoma treated with uniform chemotherapy protocol without high dose methotrexate: A single-center experience of 237 patients. J Surg Oncol 2015;112:662-8.

10. Bajpai J, Gamanagatti S, Sharma MC, Kumar R, Vishnubhatla S, Khan SA, et al. Noninvasive imaging surrogate of angiogenesis in osteosarcoma. Pediatr Blood Cancer 2010;54:526-31.

11. Hasakova K, Vician M, Reis R, Zeman M, Herichova I. Sex-dependent correlation between survival and expression of genes related to the circadian oscillator in patients with colorectal cancer. Chronobiol Int 2018;35:1423-34.

12. Wiemann B, Starnes CO. Coley's toxins, tumor necrosis factor and cancer research: A historical perspective. Pharmacol 
Ther 1994;64:529-64.

13. Hobohm U. Fever and cancer in perspective. Cancer Immunol Immunother 2001;50:391-6.

14. Bielack SS, Smeland S, Whelan JS, Marina N, Jovic G, Hook JM, et al. Methotrexate, doxorubicin, and cisplatin (MAP) plus maintenance pegylated interferon alfa- $2 b$ versus MAP alone in patients with resectable high-grade osteosarcoma and good histologic response to preoperative MAP: First results of the EURAMOS-1 good response randomized controlled trial. J Clin Oncol 2015;33:2279-87.

15. Saraf AJ, Fenger JM, Roberts RD. Osteosarcoma: Accelerating progress makes for a hopeful future. Front Oncol 2018;8:4.
This is an open access journal, and articles are distributed under the terms of the Creative Commons Attribution-NonCommercial-ShareAlike 4.0 License, which allows others to remix, tweak, and build upon the work non-commercially, as long as appropriate credit is given and the new creations are licensed under the identical terms.

\begin{tabular}{|l|l|}
\hline \multicolumn{2}{|c|}{ Access this article online } \\
\hline Quick Response Code: & Website: \\
& www.ijmpo.org \\
\cline { 2 - 2 } & Dol: \\
\hline
\end{tabular}

How to cite this article: Bajpai J, Chandrashekharan A, Banavali SD, Gupta S. Osteosarcoma journey in India: Each step reveals a new horizon! Indian J Med Paediatr Oncol 2020;41:4-6. 